\title{
Surface-structure-regulated cell-membrane penetration by monolayer-protected nanoparticles
}

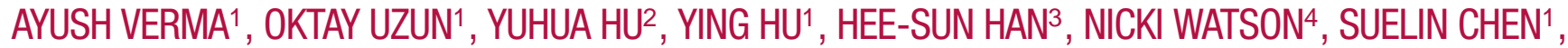 \\ DARRELL J. IRVINE ${ }^{1,5 *}$ AND FRANCESCO STELLACCI ${ }^{1 *}$
}

\author{
${ }^{1}$ Department of Materials Science and Engineering, MIT, Massachusetts 02139, USA \\ ${ }^{2}$ Department of Chemical Engineering, MIT, Massachusetts 02139, USA \\ ${ }^{3}$ Department of Chemistry, MIT, Massachusetts 02139, USA \\ ${ }^{4}$ Department of Biology, MIT, Massachusetts 02139, USA \\ ${ }^{5}$ Department of Biological Engineering, MIT, Massachusetts 02139, USA \\ *e-mail: djirvine@mit.edu; frstella@mit.edu
}

\begin{abstract}
Nanoscale objects are typically internalized by cells into membrane-bounded endosomes and fail to access the cytosolic cell machinery. Whereas some biomacromolecules may penetrate or fuse with cell membranes without overt membrane disruption, no synthetic material of comparable size has shown this property yet. Cationic nano-objects pass through cell membranes by generating transient holes, a process associated with cytotoxicity. Studies aimed at generating cell-penetrating nanomaterials have focused on the effect of size, shape and composition. Here, we compare membrane penetration by two nanoparticle 'isomers' with similar composition (same hydrophobic content), one coated with subnanometre striations of alternating anionic and hydrophobic groups, and the other coated with the same moieties but in a random distribution. We show that the former particles penetrate the plasma membrane without bilayer disruption, whereas the latter are mostly trapped in endosomes. Our results offer a paradigm for analysing the fundamental problem of cell-membrane-penetrating bio- and macro-molecules.
\end{abstract}

Nanomaterials are of great interest for use in biomedicine as imaging tools ${ }^{1-3}$, phototherapy agents $\mathrm{s}^{4,5}$ and gene delivery carriers $^{6,7}$. Their interactions with cell membranes are of central importance for all such applications. For example, many drugdelivery systems are based on the transport of therapeutic agents to the cytosol or nucleus of cells by nanoparticles; efficient delivery must be achieved while avoiding cytotoxicity during passage through cell membranes to reach intracellular target compartments ${ }^{8,9}$. Indeed, membrane penetration by synthetic ${ }^{10}$ as well as by biologically derived ${ }^{11}$ molecules/particles is currently under intense investigation. Some biomacromolecules, such as cell-penetrating peptides (CPPs), may be capable of penetrating membranes without overt lipid bilayer disruption/poration ${ }^{12-15}$. Likewise, synthetic nanomaterials with very small dimensions (molecules, metal nanoclusters ${ }^{16}$, small dendrimers ${ }^{10}$ and carbon nanotubes ${ }^{17}$ ) can also pass through cell membranes. However, to the best of our knowledge, no synthetic material larger than a few nanometres in size can pass through membranes without disrupting the integrity of these biological barriers. For example, charged particles (such as cationic quantum dots or dendrimers, mostly assisted by some degree of hydrophobicity) induce transient poration of cell membranes to enter cells, a process associated with cytotoxicity ${ }^{18}$. Alternatively, nanoparticles have been designed to explicitly disrupt endolysosomal membranes to enter the cell by force ${ }^{19}$ or enter the cell aided by exogenous agents such as
CPP chaperones ${ }^{20}$. In contrast, most nanoparticles are trapped in endosomes ${ }^{21}$ and hence do not reach the cytosol.

The surface properties of nanomaterials play a critical role in determining the outcome of their interactions with cells $\mathrm{s}^{22}$. Recently, we found that when gold nanoparticles are coated with binary mixtures of hydrophobic and hydrophilic organic molecules, ribbon-like domains of alternating composition spontaneously form in the ligand shell ${ }^{23-25}$. These domains are on average less than $6 \AA$ wide, which is of the order of or even smaller than the distribution of chemical functionalities on biomolecules such as proteins and peptides. Order, rather than random organization of the functional groups, on these particle surfaces leads to unexpected surface properties due to the molecularly close apposition of hydrophilic and hydrophobic moieties at all points on the particle surfaces: the particles exhibit unusual solubility trends and low protein binding even with high contents of hydrophobic groups $^{23,24}$. With the ordered amphiphilic structure of some CPPs in mind, we explored the interaction of these nanoparticles with living cells. Here, we show that $\sim 6 \mathrm{~nm}$ nanoparticles, coated with a shell of hydrophobic and anionic ligands regularly arranged in ribbon-like domains of alternating composition (Fig. 1a), penetrate cell membranes at $37^{\circ} \mathrm{C}$ and $4{ }^{\circ} \mathrm{C}$ without evidence of membrane disruption. Particles with identical hydrophobic content but lacking structural order in the ligand shell for the most part do not penetrate cell membranes; hence, we conclude that the 
structural organization of surface chemical groups plays a key role in regulating cell-membrane penetration.

To create amphiphilic nanoparticles, we chose gold nanoparticles $^{26}$ protected by a self-assembled monolayer of organic ligands known to determine the particles' interactions with the external environment. These particles can resemble biomolecules and biomolecular assemblies in terms of size and chemical composition and sometimes function (for example, enzymatic activity) ${ }^{27,28}$, but their tightly packed ligand shell ${ }^{24}$ lacks the structural flexibility of proteins or peptide assemblies and instead is composed of an ordered layer of surface functional groups. Simple reactions enable the synthesis of these particles coated with single- as well as multicomponent ligand shells, the composition and morphology of which can be easily and precisely controlled ${ }^{29}$. Using the unique physical chemistry offered by this system, we created water-soluble particles with ordered or disordered amphiphilic ligand shell $s^{30}$ to investigate cell-membrane penetration of nanoparticles as a function of the spatial distribution of chemical groups.

Four types of ligand-coated gold nanoparticle were synthesized $^{31}$ and studied (Table 1): particles were coated with
(1) 11-mercapto-1-undecanesulphonate (MUS), (2) a 2:1 molar mixture of MUS and 1-octanethiol (OT) (hereafter referred to as 66-34 OT), (3) a 1:2 molar mixture of MUS and OT (34-66 OT) and (4) a 2:1 molar mixture MUS: 3,7 dimethyl octane 1-thiol (br-OT) (66-34 br-OT), respectively. We used sulphonate groups because they imparted remarkable water solubility to all of the particles studied irrespective of the fraction of hydrophobic ligands used, with saturation concentrations in water in excess of $250 \mathrm{mg} \mathrm{ml}^{-1}$ (ref. 30), orders of magnitude larger than the concentrations used here. ${ }^{1} \mathrm{H}$ NMR was used to confirm the absence of unbound ligands and the particle ligand-shell composition was determined after decomposing the gold core with iodine ${ }^{32}$. The size distributions, ligand-shell packing densities and zeta potentials of the four particles studied had negligible differences (Table 1) (see the Supplementary Information). Thus, these particles had nearly identical physical characteristics, except for the composition and structure of the ligand shell, as determined by extensive scanning tunnelling microscopy (STM) studies (see the Supplementary Information) and schematically shown in Table 1. MUS particles had a homogeneous hydrophilic ligand shell, whereas 34-66 OT and 66-34 OT particles had a hydrophilic-hydrophobic striated

Table 1 Chemical, physical and morphological properties of the four particles used in this study.

\begin{tabular}{lllll}
\hline Nanoparticles & $\begin{array}{l}\text { Ligand shell } \\
\text { composition* }\end{array}$ & $\begin{array}{l}\text { Core size } \\
(\mathrm{nm})\end{array}$ & $\begin{array}{l}\text { Thermogravimetric } \\
\text { analysis }\end{array}$ & $\begin{array}{l}\zeta \text { Potential } \\
(\mathrm{mV})\end{array}$
\end{tabular}

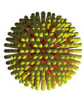

$\begin{array}{lllll}66-34 \mathrm{br}-0 \mathrm{~T} & 67 \% \text { MUS } & 4.3 \pm 1.2 & 13 & -31.1 \pm 0.73\end{array}$

Unstructured

66-34 0T


a

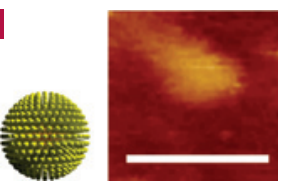

b

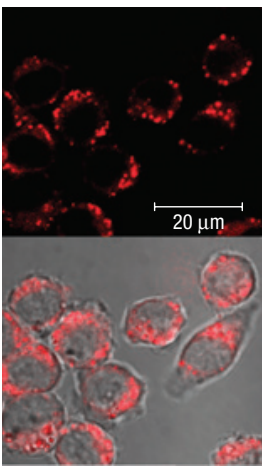

e

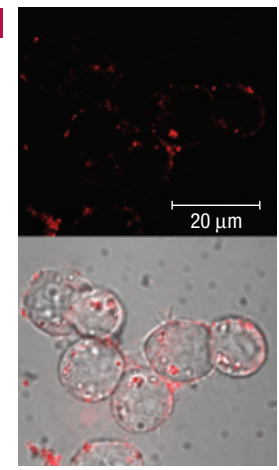

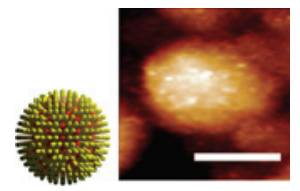

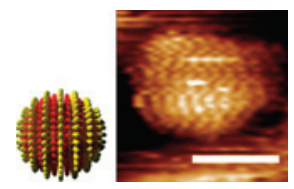

G

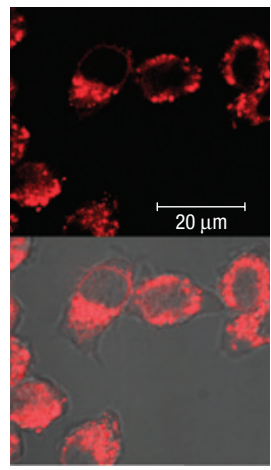

d

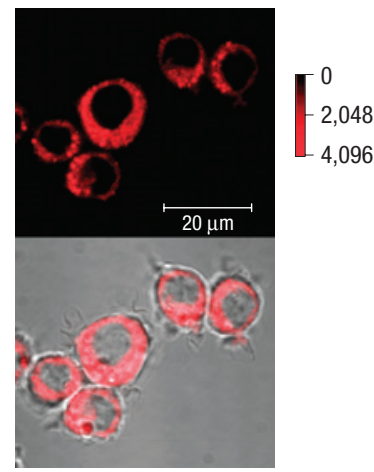

f

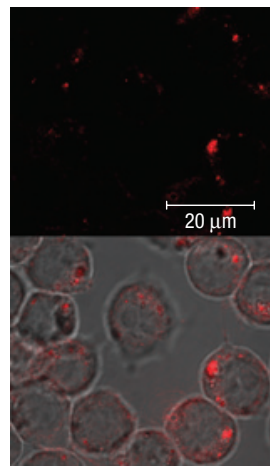

g

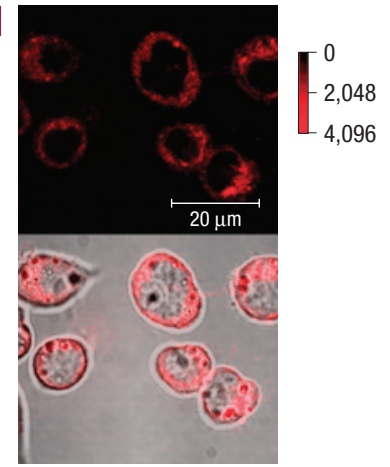

h

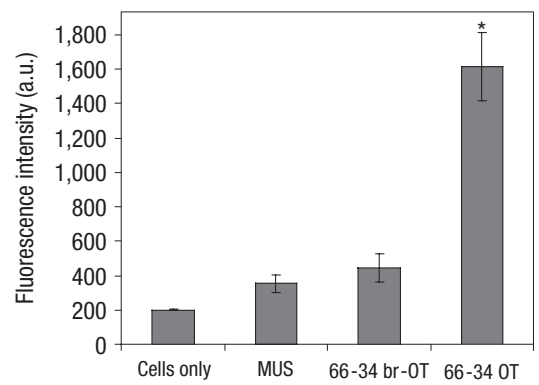

Figure 1 Nanoparticles with ordered arrangements of hydrophilic and hydrophobic surface functional groups exhibit altered patterns of subcellular localization in cells. a, Schematic diagrams of the ligand shell structure of the nanoparticles and representative STM images (scale bars $5 \mathrm{~nm}$ ). $\mathbf{b}-\mathbf{g}$, BODIPY fluorescence (upper panels with an intensity scale bar (a.u.)) and brightfield/fluorescence overlay (lower panels) images of dendritic cells incubated with $0.2 \mathrm{mg} \mathrm{ml}^{-1}$ of MUS (b,e), $66-34$ br-0T (c,f) or 66-34 0T (d,g) nanoparticles for $3 \mathrm{~h}$ in serum-free medium at $37^{\circ} \mathrm{C}(\mathbf{b}-\mathbf{d})$ or $4^{\circ} \mathrm{C}(\mathbf{e}-\mathbf{g})$. $\mathbf{h}$, Mean fluorescence intensities from confocal images at $4{ }^{\circ} \mathrm{C}$ for the different nanoparticles. *Statistically different from MUS and 66-34 br-0T $(P<0.006)$.

ligand shell; 66-34 br-OT particles had a hydrophilic-hydrophobic disordered ligand shell. The last two particles had nearly identical hydrophilic to hydrophobic ratios, the key difference residing in the ordering of hydrophilic and hydrophobic moieties of the ligand shell, that is, they can be considered as nanoscale 'isomers'.

To determine how the nature and organization of functional groups at the surface of these nanoparticles influenced their interactions with cell membranes, we labelled the particles with an average upper limit of 14 molecules of thiolated BODIPY dye (BODIPY-SH) per nanoparticle (see the Supplementary Information) and carried out confocal fluorescence microscopy studies of their uptake and intracellular distribution in living cells. A mouse dendritic cell clone DC2.4 (ref. 33) was used for these studies, representing a key class of phagocytes guarding all peripheral tissues and particularly the skin, lungs and gut mucosa. Dendritic cells are professional antigen-presenting cells, and actively sample particles/fluid/macromolecules from their environment (through phagocytosis, macropinocytosis and endocytosis) as part of their normal function. Macropinocytosis, an actin-dependent process where the cell folds membrane around a small volume of fluid, enables these cells to even internalize particles that have no interaction with the cell membrane into intracellular vesicles. To avoid potential artefacts of cell fixation ${ }^{34}$, cells were imaged live in a temperature-controlled environmental chamber. Dendritic cells were incubated with nanoparticles $\left(0.2 \mathrm{mg} \mathrm{ml}^{-1}\right)$ in serum-containing or serum-free medium for $3 \mathrm{~h}$, washed and imaged live by confocal microscopy. Figure 1 shows the difference in behaviour between the various types of nanoparticle. MUS nanoparticles bearing only the hydrophilic sulphonate ligand were internalized by dendritic cells at $37^{\circ} \mathrm{C}$ and exhibited punctate fluorescence patterns indicative of endosomal uptake (Fig. 1b), in agreement with previous findings for most types of synthetic nanoparticle ${ }^{21,35,36} .66-34$ br-OT nanoparticles bearing a disordered mixed monolayer with sulphonate and methyl headgroups also showed a punctate distribution in dendritic cells, although a low level of background fluorescence in the cytosol of some cells was also detectable (Fig. 1c). In contrast, 66-34 OT nanoparticles, which have a similar ratio of surface sulphonate and methyl groups with respect to $66-34 \mathrm{br}-\mathrm{OT}$ nanoparticles but in a 'striped' nanoscale organization, were detected in cells as a diffuse pattern of intracellular fluorescence clearly overlaid on punctate sites of brighter fluorescence due to endocytosis, suggesting passage of a fraction of these particles into the cytosol (Fig. 1d). We believe, as discussed below, that 
these particles enter cells through two coexisting mechanisms, one energy dependent (endocytosis) and the other energy independent. A similar behaviour was observed for 34-66 OT nanoparticles (see Supplementary Information, Fig. S3). Trends in nanoparticle uptake and intracellular distribution were qualitatively similar in serum-free and serum-containing medium, although a greater amount of cytosol-localized nanoparticles and fewer nanoparticles contained in vesicular structures were readily observed for the serum-free condition (see Supplementary Information, Fig. S4). The similarity in the nanoparticle uptake in serum-containing and serum-free conditions suggests 'striped' particles are resistant to non-specific protein adsorption (as previously observed in similar systems $)^{23}$, and hence retain their behaviour independent of the incubation medium. To confirm this assumption, we used dynamic light scattering to evaluate particle sizes in solution before and after incubation in serum-containing medium. The radii obtained after incubation in serum-containing medium were all in good agreement with transmission electron microscope (TEM) core size data, further confirming that the particles were well dispersed in solution. The particles were then incubated in serum-containing medium and separated from unbound proteins using gel filtration chromatography. The gel filtration chromatography traces showed that a large fraction of the 66-34 OT nanoparticles retained their original size after the incubation, whereas the 66-34 br-OT and the MUS particles showed an increase in particle size (data not shown). In fact, the dynamic light scattering data of the 'purified' particles following serum exposure showed an increase in size for the non-striped particles and almost no change for the 'striped' ones. The increase in size for the non-striped particles must be ascribed to non-specific protein adsorption, as TEM studies after incubation show nearly identical core size distribution (see Table 1, Supplementary Information).

To further explore the mechanism by which these particles entered the cells, we repeated these internalization experiments at $4{ }^{\circ} \mathrm{C}$ to inhibit mechanisms of active uptake by the cells. At this temperature, endocytic/pinocytic uptake of tracer molecules such as calcein or labelled dextrans is completely blocked in DC2.4 cells ${ }^{19}$. BODIPY channel fluorescence following the incubation of cells with MUS or 66-34 br-OT particles at $4{ }^{\circ} \mathrm{C}$ (Fig. 1e and $f$, respectively) was indistinguishable from the low levels of autofluorescence observed within untreated control cells (see Supplementary Information, Fig. S5a), whereas 66-34 OT (Fig. 1g) and 34-66 OT nanoparticles (see Supplementary Information, Fig. S5e) still seemed to enter cells at substantial levels, taking on a diffuse pattern of fluorescence consistent with cytosolic localization. A quantitative comparison of cell-associated fluorescence at $4{ }^{\circ} \mathrm{C}$ is shown in Fig. $1 \mathrm{~h}$. The fluorescence of cells incubated with MUS or 66-34 br-OT particles was not statistically different from the background autofluorescence of untreated cells, whereas the fluorescence of cells treated with 'striped' nanoparticles was eightfold greater than the background. All of the nanoparticles studied here were uniformly excluded from the nucleus of cells at both $4{ }^{\circ} \mathrm{C}$ and $37^{\circ} \mathrm{C}$. The entry of these particles into the cytosol under conditions where endocytic processes are blocked suggests that the 'striped' nanoparticles are capable of directly passing through the plasma membrane of the cells, whereas 66-34 br-OT nanoparticles with an identical ratio of hydrophilic/hydrophobic moieties but lacking the ordered arrangement of these groups do not penetrate the cell membrane. To further test this possibility and rule out potential changes in membrane structure/fluidity induced by incubating the cells at $4{ }^{\circ} \mathrm{C}$, we incubated dendritic cells with nanoparticles at $37^{\circ} \mathrm{C}$ in the presence of sodium azide and 2-deoxyglucose, which block active forms of uptake such as endocytosis. To confirm the efficacy of the inhibitors, dendritic cells were treated with the inhibitors and incubated with calcein, a small-molecule membrane-impermeable fluorophore normally taken up by endocytosis/pinocytosis. After $20 \mathrm{~min}$, control dendritic cells cultured with calcein in the absence of azide/deoxyglucose exhibited readily detectable endocytic vesicles of calcein throughout confocal $z$ sections (Fig. 2a). In contrast, dendritic cells incubated with the inhibitors and calcein showed no detectable internalized dye in three-dimensional confocal $z$ sections (data not shown). When dendritic cells were incubated with both calcein and 'striped' OT:MUS particles at $37^{\circ} \mathrm{C}$ in the presence of the internalization inhibitors, nanoparticle entry into the cytosol was readily detected after $20 \mathrm{~min}$ incubation with the particles, whereas calcein was undetectable in the cells (Fig. 2b). MUS particles and 66-34 br-OT nanoparticles failed to enter dendritic cells under these conditions (data not shown). Thus, nanoparticles with an ordered surface ligand layer were found to access the cytosol at $37^{\circ} \mathrm{C}$ under conditions where no evidence for active uptake processes such as endocytosis could be detected. Finally, consistent with an internalization mechanism involving direct interaction with the plasma membrane rather than a slower endocytic/pinocytic active uptake route, kinetic experiments showed that 'striped' nanoparticles were readily detectable in cells within $5 \mathrm{~min}$ in the presence of azide/deoxyglucose (see the Supplementary Information).

Two limitations of the confocal analysis are the resolution of optical microscopy and the possibility of artefacts in the apparent subcellular distribution arising from the release of free dye from particles. Previous studies have shown that organic thiol ligands can be displaced from gold nanoparticles by exchange reactions with cytosolic glutathione ${ }^{37}$, which is the most abundant intracellular thiol $^{38}$. This effect seems unlikely given that glutathione-mediated displacement has been shown to require timescales of the order of days ${ }^{39}$, compared with the present experiments carried out over $3 \mathrm{~h}$. However, to unequivocally interpret the confocal images and also to rule out any possible effect of the fluorophore molecules attached to the nanoparticles, we carried out an extensive TEM analysis of both fluorescently labelled and unlabelled nanoparticles taken up in cells. DC2.4 cells were incubated for $3 \mathrm{~h}$ with nanoparticles as before, washed, fixed and sectioned for TEM imaging (see the Supplementary Information). Several observations resulted from analysis of TEM images. No significant difference in overall trends was observed for particles either labelled with or without BODIPY-SH (see Supplementary Information, Fig. S14). The intracellular distribution of each of the four types of ligand-coated nanoparticle studied was in qualitative agreement with the trends observed by confocal microscopy, as illustrated by the series of TEM images in Fig. 3 for the case of unlabelled particles. MUS nanoparticles were almost exclusively found in endosomal compartments (Fig. 3a); a similar distribution was observed for 66-34 br-OT nanoparticles (Fig. 3b). 'Striped' nanoparticles, in contrast, were readily detected outside defined membrane-enclosed compartments (Fig. 3c). As shown in Fig. 3d, an enumeration of the number of free particles per $\mu \mathrm{m}^{3}$ localized outside membrane-enclosed compartments revealed a higher density of cytosolic 66-34 and 34-66 OT (see Supplementary Information, Fig. S15) nanoparticles as compared with the MUS and 66-34 br-OT particles. Interestingly, TEM images and examination of the number of cytosol-localized particles per $\mu \mathrm{m}^{3}$ obtained from the serum-containing condition showed similar trends, suggesting that the differences in particle behaviour are not mediated by differentially adsorbed serum proteins ${ }^{40}$ (see Supplementary Information, Fig. S13). The high-resolution TEM images in Fig. 4 show nanoparticles very close to the cell membrane, probably frozen 'in the act' of passing through it.

Previous studies have shown that some cationic nanoparticles and macromolecules penetrate the plasma membrane of cells by 
a
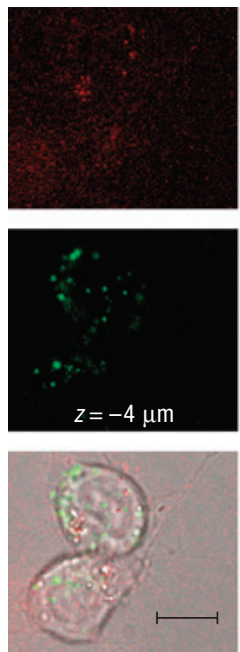

b
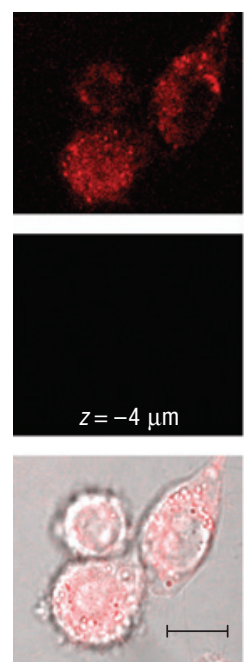
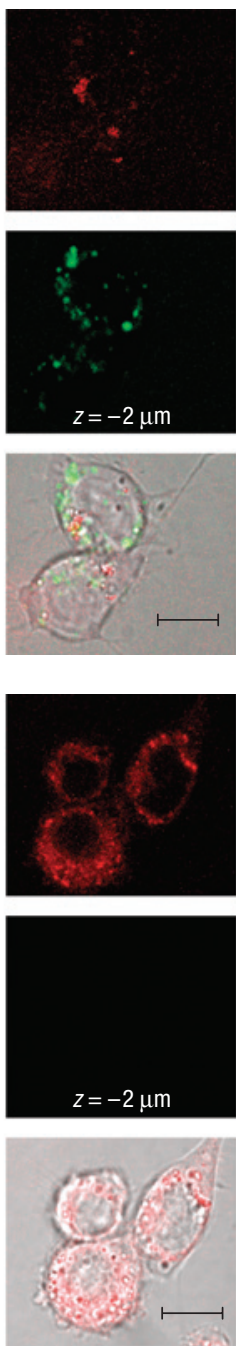
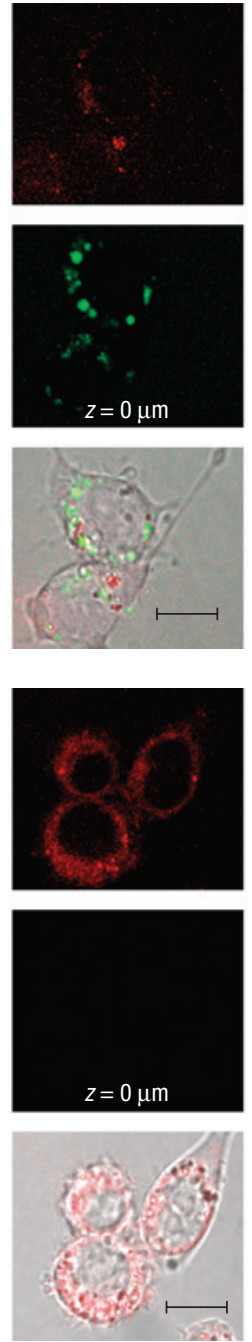
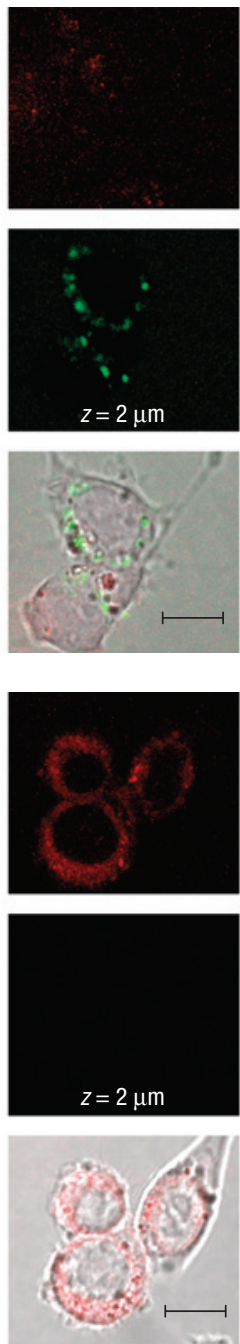
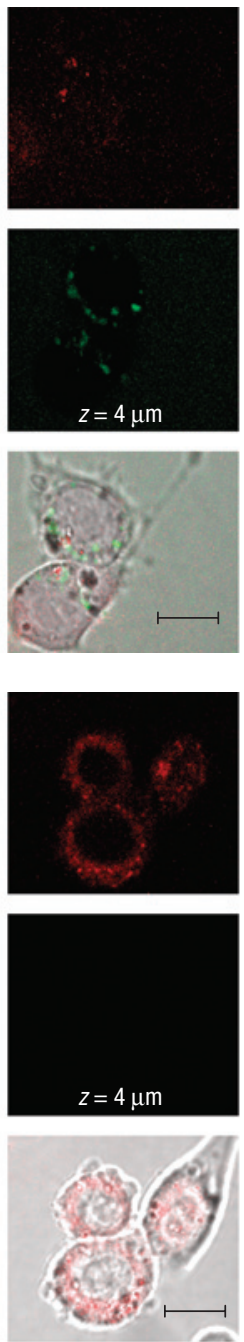
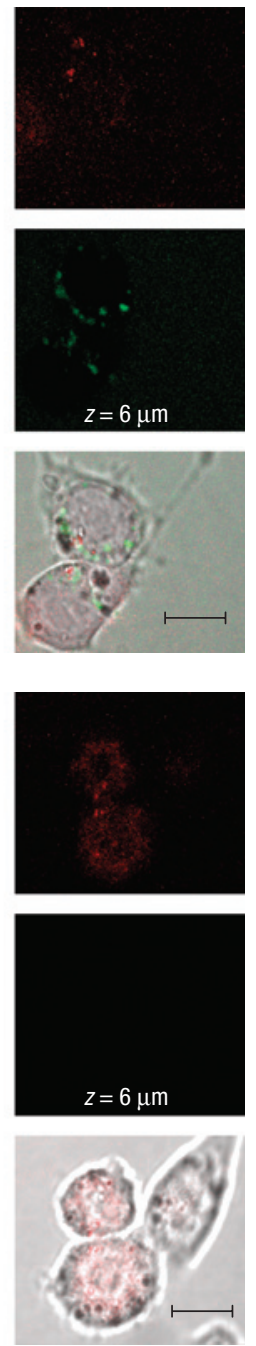

Figure 2 'Striped' nanoparticles enter cells at $37^{\circ} \mathrm{C}$ under conditions where active internalization processes are blocked. a,b, dendritic cells were incubated for $20 \mathrm{~min}$ at $37^{\circ} \mathrm{C}$ in serum-free medium with calcein (a) or calcein and 66-34 0T nanoparticles in the presence of azide and 2-deoxyglucose to block active internalization (b). Confocal optical $z$ sections in $2 \mu \mathrm{m}$ intervals taken through the midplane of cells are shown. The upper panels show nanoparticle fluorescence (red), the middle panels show calcein fluorescence (green) and the lower panels show fluorescence/brightfield overlays. Scale bars: $10 \mu \mathrm{m}$.

generating transient holes ${ }^{10}$; in contrast, some data suggest that a subset of biological membrane-penetrating macromolecules (for example, CPPs) may be capable of penetrating membranes without causing overt membrane poration ${ }^{12-15}$. The former mechanism can lead to cell death due to loss of membrane polarization and/or leakage of ions and molecules into/out of the cell. To establish whether 'striped' nanoparticles penetrate cell membranes through creation of transient holes, we tested whether the internalization of ligand-protected nanoparticles was accompanied by escape of cytosol-localized tracer dye or conversely, cytosolic entry of an initially extracellular tracer dye $e^{41,42}$. First, DC2.4 cells were co-incubated with nanoparticles and calcein; calcein remains in endolysosomal compartments in cells unless it is co-internalized with a membrane-disrupting agent ${ }^{43}$. Cells were incubated for $3 \mathrm{~h}$ with calcein and nanoparticles, washed and imaged live by confocal microscopy; the localization of calcein in individual cells was then scored (endosomal versus cytosolic). As a positive control, dendritic cells were incubated with cationic gold nanoparticles bearing 11-mercaptoundecane-tetramethylammonium chloride (TMA) ligands, which would be expected to disrupt cell membranes on the basis of previously published work ${ }^{10}$. As shown in Fig. 5a,c, TMA nanoparticles caused calcein to escape to the cytosol of $\sim 50 \%$ of the dendritic cells, whereas dendritic cells incubated with calcein alone exhibited a solely punctate endosomal distribution of dye. Strikingly, 66-34 OT (Fig. 5a,b) and 34-66 OT nanoparticles (see Supplementary Information, Fig. S6) (both fluorescently labelled and unlabelled) did not promote calcein entry into the cytosol, despite the data described above showing that these particles clearly access the cytosolic compartment. We next tested whether 'striped' nanoparticles caused an escape of tracer molecules from the cytosol of cells during uptake: dendritic cells were pre-loaded with calcein-AM, an acetomethoxy form of the dye that is trapped in the cytosol by conversion from a membrane-permeable to membrane-impermeable form by intracellular esterases. Again, 66-34 OT particles entered cells without any measurable leakage of calcein from the cells (see Supplementary Information, Fig. S7). To further, albeit indirectly, confirm that 'striated' particles penetrate cell membranes without causing substantial 

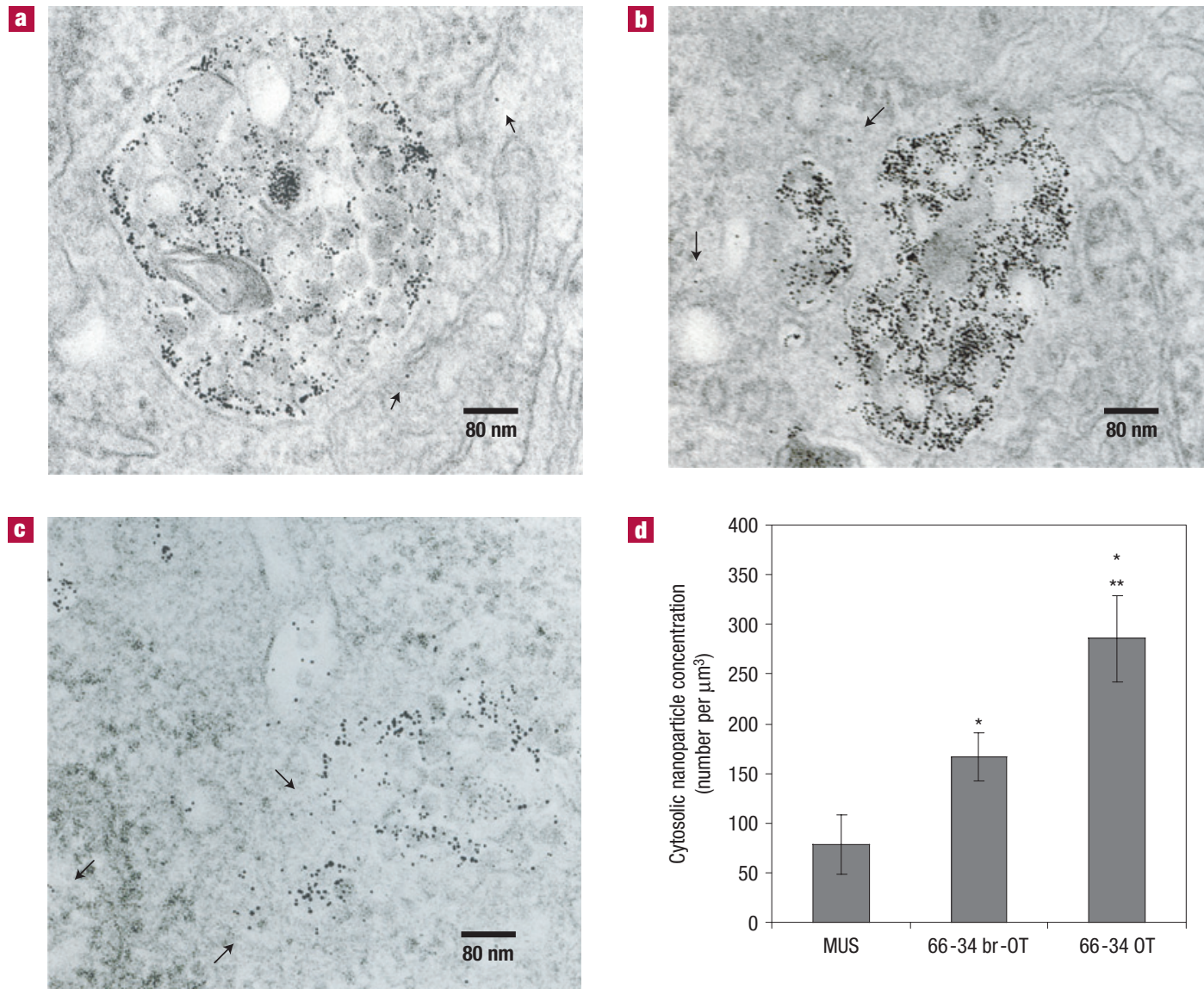

Figure 3 Nanoparticles with sulphonate-only or disordered sulphonate/methyl-group surfaces are largely confined to endosomal compartments, whereas 'striped' sulphonate/methyl-group-bearing nanoparticles enter the cytosol. a-c, TEM images of DC2.4 cells following incubation with MUS (a), 66-34 br-0T (b) or 66-34 0T (c) nanoparticles for $3 \mathrm{~h}$ in serum-free medium. Free nanoparticles are denoted by arrows. $\mathbf{d}$, Number of cytosolic nanoparticles per $\mu \mathrm{m}^{3}$ ( \pm s.d.), determined by scoring the localization of $>6,500$ particles per condition. ${ }^{*}$ Statistically different from MUS $(P<0.005) .{ }^{*}$ Statistically different from $66-34 \mathrm{br}-0 \mathrm{~T}(P<0.005)$.
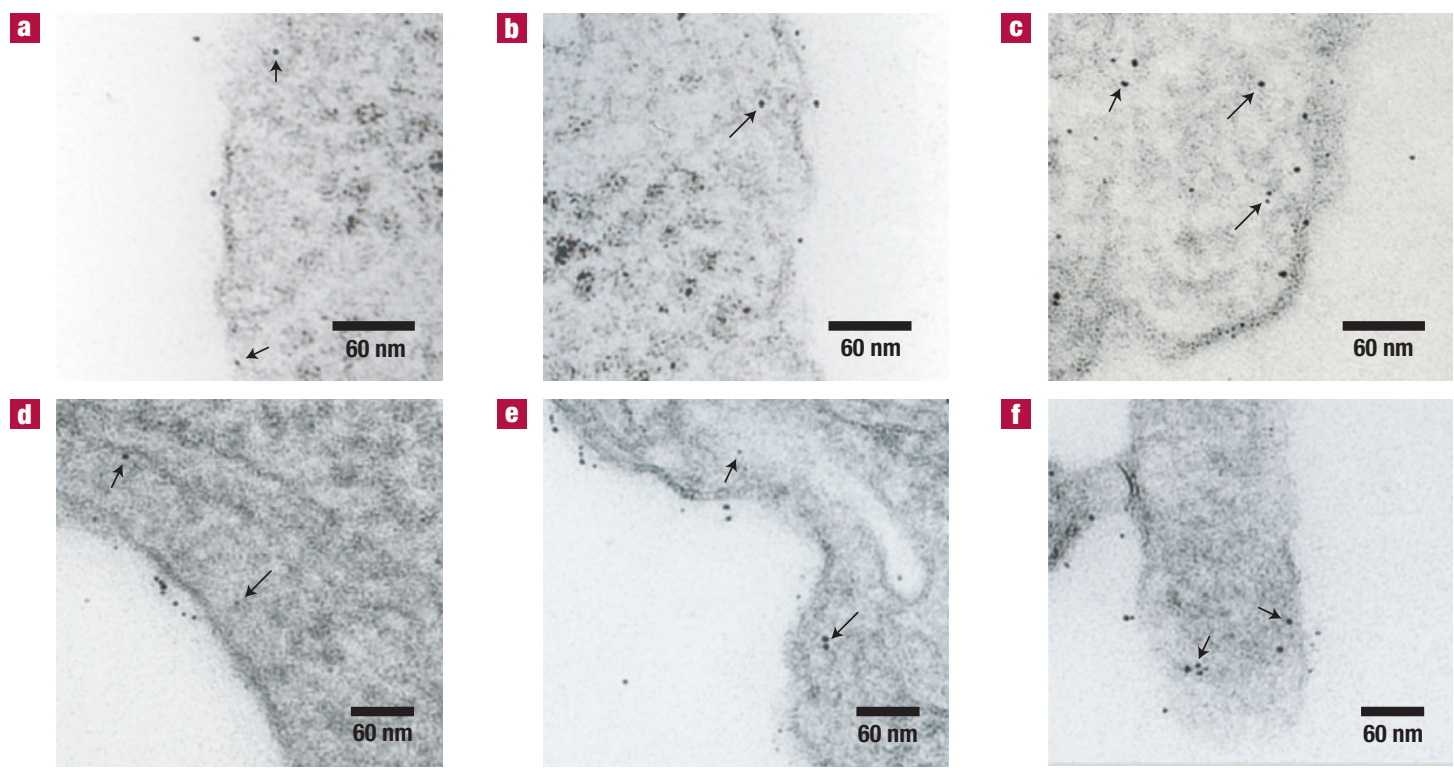

Figure 4 'Striped' particles visualized at different stages of crossing the cell membrane of dendritic cells. a-f, TEM images of 66-34 0T (a-c) and 34-66 0T (d-f) nanoparticles incubated with cells in serum-free medium at $37^{\circ} \mathrm{C}$. The arrows indicate a few of the particles seen intracellular to the cell membrane. 


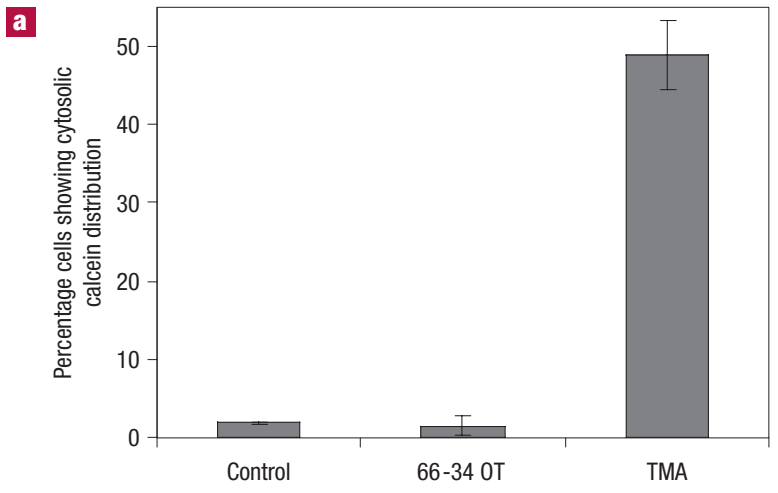

b
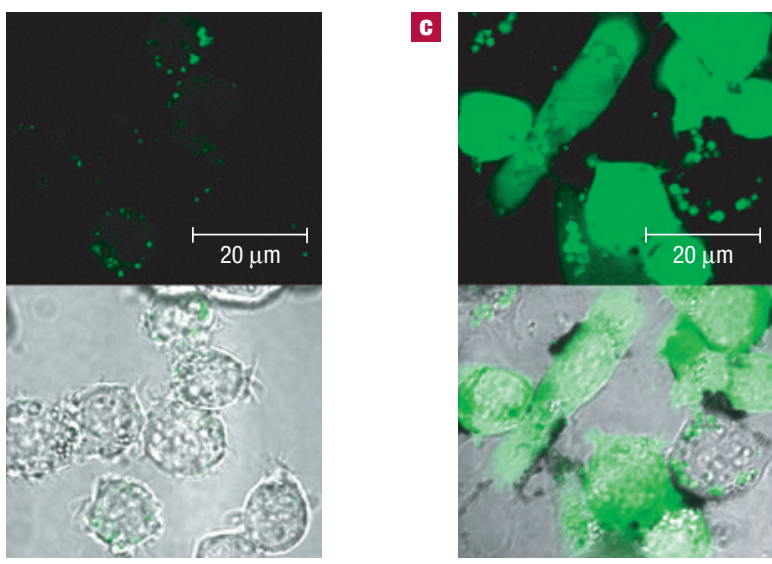

Figure 5 'Striped' nanoparticles penetrate cell membranes without generating transient holes, in contrast with the known behaviour of cationic nanoparticles. a, Percentage of total cells showing cytosolic distribution of calcein. At least 100 cells were sampled for each particle and the control. b,c, Confocal images showing calcein uptake and intracellular distribution in the presence of 66-34 0T (b) or TMA (c) nanoparticles.

membrane disruption, we measured their cytotoxicity relative to cationic TMA-coated particles using a colony-forming assay. DC2.4 cells were incubated with nanoparticles for $3 \mathrm{~h}$, washed, replated at equal densities and allowed to grow for $24 \mathrm{~h}$. We found that cells treated with 66-34 and 34-66 OT nanoparticles survived/proliferated at a level that was $\sim 90 \%$ of that of untreated control cells, whereas only $34 \%$ were recovered following treatment with TMA-coated particles (see Supplementary Information, Fig. S10). Similar results were obtained when studying immediate toxicity of cells with propidium iodide staining and MTT (3-(4,5-dimethylthiazol-2-yl)-2,5-diphenyltetrazolium bromide) assay (see Supplementary Information, Figs S11,S12).

In these studies, we consistently observed no nanoparticle nuclear uptake, as well as no evidence for escape of nanoparticles from cells for up to $6 \mathrm{~h}$ after internalization occurred (data not shown). We speculate that these two phenomena could be related; it is possible that when particles are in the cytosol they lose the delicate compositional balance and structural motif that is needed to permeate cell membranes. However, given the unique structure of the nuclear double membrane, further studies need to be carried out to fully understand these observations. Moreover, to test the generality of our results, we repeated $37^{\circ} \mathrm{C}$ and $4{ }^{\circ} \mathrm{C}$ uptake experiments with a second common cell line, mouse embryonic fibroblasts. The same trends in particle uptake, with 'striped' particles accessing the cytosol, were observed (see Supplementary Information, Fig. S9).

Our results highlight the importance of structure in the interactions of nanoparticles with cell membranes. We synthesized two nanoparticles that could be loosely characterized as the nanoscale equivalent of chemical isomers. They have the same size and shape, and are coated with the same ratio of hydrophobic to hydrophilic molecules, the latter being identical (MUS) whereas the former differing slightly (OT versus br-OT) to achieve different ligand shell structures. These two particles (66-34 OT and 66-34 br-OT) showed very similar solubility and wetting behaviour as highlighted by nearly identical zeta potentials. They differed only in the molecular arrangement of surface chemical groups: 66-34 OT exhibiting a striated ligand organization and 66-34 br-OT lacking this structured arrangement. This seemingly small difference led to drastically different behaviour in cell-membrane-penetration properties. Confocal studies of cell uptake of these particles (dye functionalized to the same extent) in DC2.4 cells at $37^{\circ} \mathrm{C}$ (in both serum-containing and serum-free conditions) showed a pronounced punctate fluorescence distribution pattern indicative of endosomal entrapment for 66-34 br-OT nanoparticles, whereas 66-34 OT particles exhibited a more diffuse fluorescence pattern indicating escape to the cytosol. These differences in intracellular localization were confirmed through TEM studies, which were also used to rule out any effect of the presence of the dye in the ligand shell. Nanoparticle uptake experiments at $4{ }^{\circ} \mathrm{C}$ were used to prove that the 66-34 OT nanoparticles can directly pass through cell membranes and do not require internalization by active endocytosis or pinocytosis to reach the cytosol, whereas at $4{ }^{\circ} \mathrm{C}$ 66-34 br-OT nanoparticles are almost completely blocked from cell entry. This was also confirmed by detection of nanoparticle entry into cells within $5 \mathrm{~min}$ despite actively blocking endocytosis by treating the cells with sodium azide and 2-deoxyglucose (marked by no calcein uptake). Finally, we determined that 66-34 OT nanoparticles pass through cell membranes without causing overt membrane poration through studies with tracer dyes (no noticeable leakage) and cytotoxicity assays (minimal toxicity). This is in stark contrast with cationic nanoparticles, which are known to penetrate cell membranes by creating pores ${ }^{10}$ and induce cytotoxicity ${ }^{18}$. At the moment we can only speculate on the mechanism that leads to cell-membrane penetration. A possible explanation is that the 'rigid' arrangement of the amphiphilic domains on the nanoparticles permits non-disruptive fusion of the nanoparticle with cell membranes (a more fluid mixed layer) and subsequent penetration through the bilayer, in a manner reminiscent of some models for entry of CPPs.

In summary, we believe that by highlighting the role of ligand structure and order in cell-membrane penetration by nanoparticles, this work will shed new light on the understanding of the molecular interactions that lead to cell-membrane permeation, certainly on nanoparticles, possibly on larger organic particles and hopefully in CPPs. Future work, besides the fundamental studies highlighted above, includes the engineering of novel and more efficient low-toxicity drug-delivery systems based on the design rules for membrane penetration identified here and on the analysis of potential safety issues associated with synthetic nanomaterials ${ }^{44}$.

Received 4 December 2007; accepted 23 April 2008; published 25 May 2008

\section{References}

1. Weissleder, R. Molecular imaging in cancer. Science 312, 1168-1171 (2006).

2. Somers, R. C., Bawendi, M. G. \& Nocera, D. G. CdSe nanocrystal based chem-/bio-sensors. Chem. Soc. Rev. 36, 579-591 (2007).

3. Lewin, M. et al. Tat peptide-derivatized magnetic nanoparticles allow in vivo tracking and recovery of progenitor cells. Nature Biotechnol. 18, 410-414 (2000)

4. West, J. L. \& Halas, N. J. Engineered nanomaterials for biophotonics applications: Improving sensing, imaging, and therapeutics. Annu. Rev. Biomed. Eng. 5, 285-292 (2003). 
5. El-Sayed, I. H., Huang, X. H. \& El-Sayed, M. A. Selective laser photo-thermal therapy of epithelial carcinoma using anti-EGFR antibody conjugated gold nanoparticles. Cancer Lett. 239, 129-135 (2006)

6. Rosi, N. L. et al. Oligonucleotide-modified gold nanoparticles for intracellular gene regulation Science 312, 1027-1030 (2006)

7. Han, G. et al. Light-regulated release of DNA and its delivery to nuclei by means of photolabile gold nanoparticles. Angew. Chem. Int. Ed. 45, 3165-3169 (2006).

8. Allen, T. M. \& Cullis, P. R. Drug delivery systems: Entering the mainstream. Science $\mathbf{3 0 3}$ 1818-1822 (2004)

9. Choleris, E. et al. Microparticle-based delivery of oxytocin receptor antisense DNA in the medial amygdala blocks social recognition in female mice. Proc. Natl Acad. Sci. USA 104, 4670-4675 (2007).

10. Leroueil, P. R. et al. Nanoparticle interaction with biological membranes: Does nanotechnology present a janus face? Acc. Chem. Res. 40, 335-342 (2007).

11. Zorko, M. \& Langel, U. Cell-penetrating peptides: mechanism and kinetics of cargo delivery. Adv. Drug Deliv. Rev. 57, 529-545 (2005).

12. Herbig, M. E., Assi, F., Textor, M. \& Merkle, H. P. The cell penetrating peptides pVEC and W2-pVEC induce transformation of gel phase domains in phospholipid bilayers without affecting their integrity. Biochemistry 45, 3598-3609 (2006)

13. Takeuchi, T. et al. Direct and rapid cytosolic delivery using cell-penetrating peptides mediated by pyrenebutyrate. ACS Chem. Biol. 1, 299-303 (2006).

14. Thoren, P. E. G. et al. Uptake of analogs of penetratin, Tat(48-60) and oligoarginine in live cells. Biochem. Biophys. Res. Commun. 307, 100-107 (2003).

15. Patel, L. N., Zaro, J. L. \& Shen, W. C. Cell penetrating peptides: Intracellular pathways and pharmaceutical perspectives. Pharm. Res. 24, 1977-1992 (2007).

16. Yu, J., Patel, S. A. \& Dickson, R. M. In vitro and intracellular production of peptide-encapsulated fluorescent silver nanoclusters. Angew. Chem. Int. Ed. 46, 2028-2030 (2007).

17. Kostarelos, K. et al. Cellular uptake of functionalized carbon nanotubes is independent of functional group and cell type. Nature Nanotechnol. 2, 108-113 (2007).

18. Lovric, J. et al. Differences in subcellular distribution and toxicity of green and red emitting CdTe quantum dots. J. Mol. Med. 83, 377-385 (2005).

19. Hu, Y. et al. Cytosolic delivery of membrane-impermeable molecules in dendritic cells using pH-responsive core-shell nanoparticles. Nano Lett. 7, 3056-3064 (2007).

20. Tkachenko, A. G. et al. Multifunctional gold nanoparticle-peptide complexes for nuclear targeting. J. Am. Chem. Soc. 125, 4700-4701 (2003).

21. Shukla, R. et al. Biocompatibility of gold nanoparticles and their endocytotic fate inside the cellular compartment: A microscopic overview. Langmuir 21, 10644-10654 (2005).

22. Colvin, V. Correlating the physical and chemical properties of nanoparticles to their biological activity. Environ. Mol. Mutagen. 48, 533-533 (2007).

23. Jackson, A. M., Myerson, J. W. \& Stellacci, F. Spontaneous assembly of subnanometre-ordered domains in the ligand shell of monolayer-protected nanoparticles. Nature Mater. 3, 330-336 (2004).

24. Jackson, A. M., Hu, Y., Silva, P. J. \& Stellacci, F. From homoligand- to mixed-ligand-monolayer-protected metal nanoparticles: A scanning tunneling microscopy investigation. J. Am. Chem. Soc. 128, 11135-11149 (2006)

25. DeVries, G. A et al. Divalent metal nanoparticles. Science 315, 358-361 (2007).

26. Daniel, M. C. \& Astruc, D. Gold nanoparticles: Assembly, supramolecular chemistry, quantum-size-related properties, and applications toward biology, catalysis, and nanotechnology. Chem. Rev. 104, 293-346 (2004).

27. Manea, F., Houillon, F. B., Pasquato, L. \& Scrimin, P. Nanozymes: Gold-nanoparticle-based transphosphorylation catalysts. Angew. Chem. Int. Ed. 43, 6165-6169 (2004).

28. Kisailus, D., Najarian, M., Weaver, J. C. \& Morse, D. E. Functionalized gold nanoparticles mimic catalytic activity of a polysiloxane-synthesizing enzyme. Adv. Mater. 17, 1234 (2005).

29. Templeton, A. C., Wuelfing, M. P. \& Murray, R. W. Monolayer protected cluster molecules. Acc. Chem. Res. 33, 27-36 (2000).
30. Uzun, O. et al. Water-soluble amphiphilic gold nanoparticles with structured ligand shells. Chem. Commun. 196-198 (2008)

31. Kang, S. Y. \& Kim, K. Comparative study of dodecanethiol-derivatized silver nanoparticles prepared in one-phase and two-phase systems. Langmuir 14, 226-230 (1998).

32. Tan, H., Zhan, T. \& Fan, W. Y. Direct functionalization of the hydroxyl group of the 6-mercapto-1-hexanol (MCH) ligand attached to gold nanoclusters. J. Phys. Chem. B 110 21690-21693 (2006)

33. Shen, Z. H., Reznikoff, G., Dranoff, G. \& Rock, K. L. Cloned dendritic cells can present exogenous antigens on both MHC class I and class II molecules. J. Immunol. 158, 2723-2730 (1997).

34. Richard, J. P. et al. Cell-penetrating peptides-A reevaluation of the mechanism of cellular uptake. J. Biol. Chem. 278, 585-590 (2003).

35. Parak, W. J. et al. Cell motility and metastatic potential studies based on quantum dot imaging of phagokinetic tracks. Adv. Mater. 14, 882-885 (2002).

36. Wilhelm, C., Gazeau, F., Roger, J., Pons, J. N. \& Bacri, J. C. Interaction of anionic superparamagnetic nanoparticles with cells: Kinetic analyses of membrane adsorption and subsequent internalization. Langmuir 18, 8148-8155 (2002).

37. Verma, A., Simard, J. M., Worrall, J. W. E. \& Rotello, V. M. Tunable reactivation of nanoparticle-inhibited beta-galactosidase by glutathione at intracellular concentrations. J. Am. Chem. Soc. 126, 13987-13991 (2004).

38. Meister, A. \& Anderson, M. E. Glutathione. Annu. Rev. Biochem. 52, 711-760 (1983).

39. Hong, R. et al. Glutathione-mediated delivery and release using monolayer protected nanoparticle carriers. J. Am. Chem. Soc. 128, 1078-1079 (2006).

40. Cedervall, T. et al. Detailed identification of plasma proteins adsorbed on copolymer nanoparticles. Angew. Chem. Int. Ed. 46, 5754-5756 (2007).

41. Hong, S. P. et al. Interaction of polycationic polymers with supported lipid bilayers and cells Nanoscale hole formation and enhanced membrane permeability. Bioconjug. Chem. 17, 728-734 (2006).

42. Hong, S. P. et al. Interaction of poly(amidoamine) dendrimers with supported lipid bilayers and cells: Hole formation and the relation to transport. Bioconjug. Chem. 15, 774-782 (2004).

43. Zhang, L. J., Rozek, A. \& Hancock, R. E. W. Interaction of cationic antimicrobial peptides with model membranes. J. Biol. Chem. 276, 35714-35722 (2001).

44. Nel, A., Xia, T., Madler, L. \& Li, N. Toxic potential of materials at the nanolevel. Science 311 $622-627$ (2006).

Supplementary Information accompanies this paper on www.nature.com/naturematerials.

\section{Acknowledgements}

This work was supported by the National Science Foundation (CAREER Award) and the NHLB

TPEN program (U01-HL080731). F.S. is grateful to the Packard Foundation for their generous award. This paper is dedicated to A. Mayes in recognition of the mentor role she had for both corresponding authors.

\section{Author contributions}

D.J.I. and F.S. conceived the research question. A.V., O.U., D.J.I. and F.S. designed the experiments and analysed all of the data. The biological experiments were carried out by A.V. and Yu.H.; TEM experiments were carried out by A.V. and N.W., STM by Yi.H. and S.C., nanoparticle synthesis and characterization by O.U. and H.S.H. The paper was written by A.V., O.U., D.J.I. and F.S.

Author information

Reprints and permission information is available online at http://npg.nature.com/reprintsandpermissions. Correspondence and requests for materials should be addressed to D.J.I. or F.S. 